\title{
Distorção do vento na área molhada por canhões hidráulicos: Extensão da modelagem para aspersores médios $^{1}$
}

\author{
Lessandro C. Faria ${ }^{2}$, Alberto Colombo ${ }^{3}$, Henrique F. E. de Oliveira ${ }^{3}$, \\ Samuel Beskow ${ }^{2}$ \& Giuliani do Prado ${ }^{4}$
}

\begin{abstract}
RESUMO
A hipótese da existência de uma relação linear entre o alcance de canhões hidráulicos e a velocidade do vento, descrita no modelo de Richards \& Weatherhead e amplamente utilizada na simulação da distribuição de água de canhões hidráulicos em condições de vento, foi avaliada neste trabalho para aspersores de tamanho médio. Na avaliação foram consideradas distâncias de alcance entre os aspersores modelos Agropolo/NY e Naan/5024 e os limites de sua área molhada, observadas em ensaios de campo, medidos nas direções: contrária, a favor e perpendicular ao vento. As retas ajustadas pelo método dos mínimos quadrados indicaram, em relação ao alcance do aspersor quando da ausência de vento, que para cada incremento de $1 \mathrm{~m} \mathrm{~s}^{-1}$ na velocidade do vento ocorreram: (i) um decréscimo de 3,7\% na direção contrária ao vento; (ii) um decréscimo de $3,0 \%$ na direção perpendicular ao vento e (iii) um acréscimo de $2,2 \%$ na mesma direção do vento, indicando que no intervalo de velocidades de vento consideradas $\left(0\right.$ a $\left.3 \mathrm{~m} \mathrm{~s}^{-1}\right)$ a hipótese de uma relação linear entre o alcance de aspersores de tamanho médio e a velocidade do vento, foi mantida.
\end{abstract}

Palavras-chave: ensaios de uniformidade, simulação, modelo de Richard \& Weatherhead

\section{Wind distortion in wetted area of gun sprinklers: Extension of modeling to medium-sized sprinklers}

\begin{abstract}
The hypothesis of a linear relationship between wetted radius of gun sprinklers and wind velocity, as proposed by the Richards \& Weatherhead model and widely used for simulation of water distribution by gun sprinklers under windy conditions, was evaluated in this study for medium-sized sprinklers. Distances from the sprinkler models used in this study (Agropolo/NY e Naan/5024) to the limit of the wetted area were determined through various field tests for upwind, downwind and crosswind conditions. Using the least square method it was found that, in relation to the no-wind radius, for each $1 \mathrm{~m} \mathrm{~s}^{-1}$ increase in wind velocity there was: (i) a distance reduction of 3.7\% for upwind edge; (ii) a 3.0\% decrease in distance for crosswind edge; and (iii) a $2.2 \%$ increase in distance for downwind edge. These results indicated that the linear relationship found between wetted radius and wind velocity was valid for the range of wind velocities considered in this study $\left(0-3 \mathrm{~m} \mathrm{~s}^{-1}\right)$.
\end{abstract}

Key words: uniformity tests, simulation, model by Richard \& Weatherhead

\footnotetext{
Parte da Tese de Doutorado do primeiro autor, apresentada à UFLA, com bolsa da CAPES

2 CDTec/Engenharia Hídrica/UFPel, CEP 96010-610, Pelotas, RS. Fone: (53)3921-1240. E-mail: lessandro.faria@ufpel.edu.br; samuel.beskow@ufpel.edu.br

DEG/UFLA, CP 3037, CEP 37200-000, Lavras, MG. Fone: (35) 3829-1388. E-mail: acolombo@ufla.com; henrique.ellias@hotmail.com

DEA/UEM, CEP 87820-000, Cidade Gaúcha, PR. Fone: (44) 3675-1779. E-mail: gprado@uem.br
} 


\section{INTRODUÇÃO}

A uniformidade de sistemas de irrigação pode ser afetada por fatores operacionais e climáticos. Dentre os fatores operacionais, Keller \& Bliesner (1990) destacam altura de instalação do aspersor, diâmetro do bocal, pressão de serviço e ângulo de saída do jato de água, que determinam a forma geométrica do perfil radial de distribuição de água e, dentre os fatores climáticos, Carrión et al. (2001) destacam a velocidade e a direção do vento, durante a irrigação.

Dentre esses fatores o efeito do vento na distribuição de água de aspersores tem recebido atenção de diversos pesquisadores. Bernuth \& Seginer (1990) demonstraram que, devido à ação do vento, ocorre distorção no padrão de distribuição de água do aspersor, diminuindo muito na direção contrária ao vento e aumentando pouco na mesma direção do vento. De acordo com os autores, ocorre um deslocamento na mesma direção do vento, do centro de gravidade da distribuição de água do aspersor de aproximadamente $1 \mathrm{~m}$ para cada incremento de $1 \mathrm{~m} \mathrm{~s}^{-1}$ na velocidade do vento.

Em ensaios de distribuição de água sob condições de vento, com aspersores de tamanho médio, Tarjuelo et al. (1999) constataram que na direção a favor do vento ocorreu um aumento médio no alcance de $0,77 \mathrm{~m}$ (variando de 0,36 a $1,14 \mathrm{~m}$ ) em relação ao alcance sem vento do aspersor. Para os alcances contra e perpendicular ao vento, os referidos autores observaram, para cada incremento de $1 \mathrm{~m} \mathrm{~s}^{-1}$, uma redução média de 1,99 m (variando de 1,69 e 2,74 m) na direção contrária à do vento e uma redução média de 1,37 m (variando de 0,94 a 1,90 m) na direção perpendicular em relação ao alcance sem vento do aspersor.

De acordo com Oliveira et al. (2009), devido às dificuldades de se estudar os efeitos do vento sobre a uniformidade de aplicação de água dos sistemas de irrigação por aspersão em condições de campo, a modelagem computacional da distribuição de água de aspersores torna-se ferramenta muito utilizada sendo, de acordo com Prado \& Colombo (2010a), importante no auxílio das tomadas de decisões em projetos de irrigação, avaliação de sistemas de irrigação e desenvolvimento de novos aspersores.

Para aspersores de tamanho médio, tal como demonstrado por Carrión et al. (2001), Montero et al. (2001) e Playán et al. (2006), é comum a utilização de modelos físicos (baseados em equações diferenciais que descrevem a trajetória das gotas desde o momento que elas deixam o aspersor, até atingirem a superfície do solo) para prever a uniformidade de distribuição de água de aspersores. Porém, de acordo com Oliveira et al. (2009) tem-se, na utilização desses modelos, em alguns casos, dificuldades de se estabelecer valores de coeficientes de arraste, posição inicial da gota em relação ao aspersor, bem como a velocidade e o ângulo de trajetória das gotas.

Para minimizar eventuais erros possíveis de ocorrer na utilização de modelos baseados em teorias balísticas, alguns pesquisadores conduziram seus estudos utilizando modelos empíricos devido, principalmente, ao número reduzido de ensaios de campo em condições de vento necessários ao ajuste dos parâmetros e também ao reduzido número de parâmetros a serem ajustados.

Dentre os modelos empíricos o proposto por Richards \& Weatherhead (1993) tem sido muito utilizado para prever a uniformidade de distribuição de água, sob condições de vento, de canhões hidráulicos operando em sistemas autopropelidos (Granier et al., 2003; Newell, 2003; Smith et al., 2008; Prado \& Colombo, 2010a,b) e em sistemas convencionais de irrigação (Faria et al., 2009). No entanto, referidas pesquisas estão voltadas para a utilização de aspersores que operam com altas pressões (canhões hidráulicos), sem considerar a aplicabilidade deste modelo para aspersores de menor porte, como sugerido por Granier et al. (2003).

Visando preencher esta lacuna propôs-se, neste trabalho, avaliar, a partir de ensaios de campo dos aspersores modelos Agropolo/NY e Naan/5024, a hipótese da existência de uma relação linear entre o alcance de aspersores de tamanho médio e a velocidade do vento, que é assumida no modelo semiempírico de Richards \& Weatherhead (1993) e comprovada por Oliveira et al. (2009) para o canhão hidráulico Plona-RL250.

\section{Material e MÉTODOS}

Foram utilizados dados obtidos em 32 ensaios de distribuição de água dos aspersores modelos Agropolo/NY e Naan/5024 (Tabela 1), ambos com ângulo de inclinação do lançamento do jato de água de $12^{\circ}$. Os ensaios da distribuição espacial de água, que tiveram duração de uma hora, foram realizados em uma área gramada próximo ao Laboratório de Hidráulica da Universidade Federal de Lavras (UFLA), Lavras, MG (Beskow et al., 2011), seguindo a norma ISO 7749-2 (ISO, 1990).

Tabela 1. Velocidade média do vento e combinações de bocal e pressão de serviço nos ensaios de campo com aspersores Agropolo/NY e Naan/5024

\begin{tabular}{|c|c|c|c|c|c|}
\hline \multicolumn{3}{|c|}{ Agropolo/NY ( 3,5 mm) } & \multicolumn{3}{|c|}{ Naan/5024 (3,0 mm) } \\
\hline \multicolumn{6}{|c|}{ Pressão de serviço (kPa) } \\
\hline 194 & 245 & 294 & 245 & 294 & 343 \\
\hline \multicolumn{6}{|c|}{ Velocidade do vento $\left(\mathrm{m} \mathrm{s}^{-1}\right)$} \\
\hline 0,94 & 1,12 & 1,36 & 1,40 & 1,06 & 0,92 \\
\hline 1,02 & 1,17 & 1,40 & 1,46 & 1,13 & 1,12 \\
\hline 1,09 & 1,33 & 1,64 & 1,46 & 1,36 & 1,29 \\
\hline 1,37 & 1,46 & 2,01 & 2,05 & 1,68 & 2,40 \\
\hline 2,20 & 1,48 & 2,13 & - & 2,15 & - \\
\hline 2,45 & 2,08 & - & - & - & - \\
\hline 2,92 & 2,40 & - & - & - & - \\
\hline
\end{tabular}

Nos ensaios de campo, que tiveram duração de uma hora, o aspersor foi posicionado no centro de uma malha de coletores, regularmente espaçados em $2 \mathrm{~m}$, representados por uma matriz de 12 x 12, totalizando 144 coletores, contendo os valores de intensidade de precipitação $\left(\mathrm{mm} \mathrm{h}^{-1}\right)$ obtidos em coletores do tipo lata de óleo (de cozinha), com diâmetro interno de $83,5 \mathrm{~mm}$ e altura de $190 \mathrm{~mm}$, estando sua borda superior situada a 500 mm da superfície do solo.

As velocidades e direções de vento em cada ensaio de campo, medidas a $2 \mathrm{~m}$ de altura da superfície do solo, foram registradas por uma estação agrometeorológica, instalada junto à área experimental, em intervalos regulares de $5 \mathrm{~min}$.

Nas determinações experimentais das dimensões da área molhada distorcida pelo vento as 32 matrizes, contendo os dados da distribuição espacial da água observados nos ensaios 
de campo, foram transformadas em mapas de superfície (Figura 1) que mostravam, em escala apropriada, a distribuição espacial das linhas de mesma intensidade de precipitação (desde 0,25 até $9,25 \mathrm{~mm} \mathrm{~h}^{-1} \mathrm{em}$ intervalos regulares de $\left.1 \mathrm{~mm} \mathrm{~h}^{-1}\right)$.

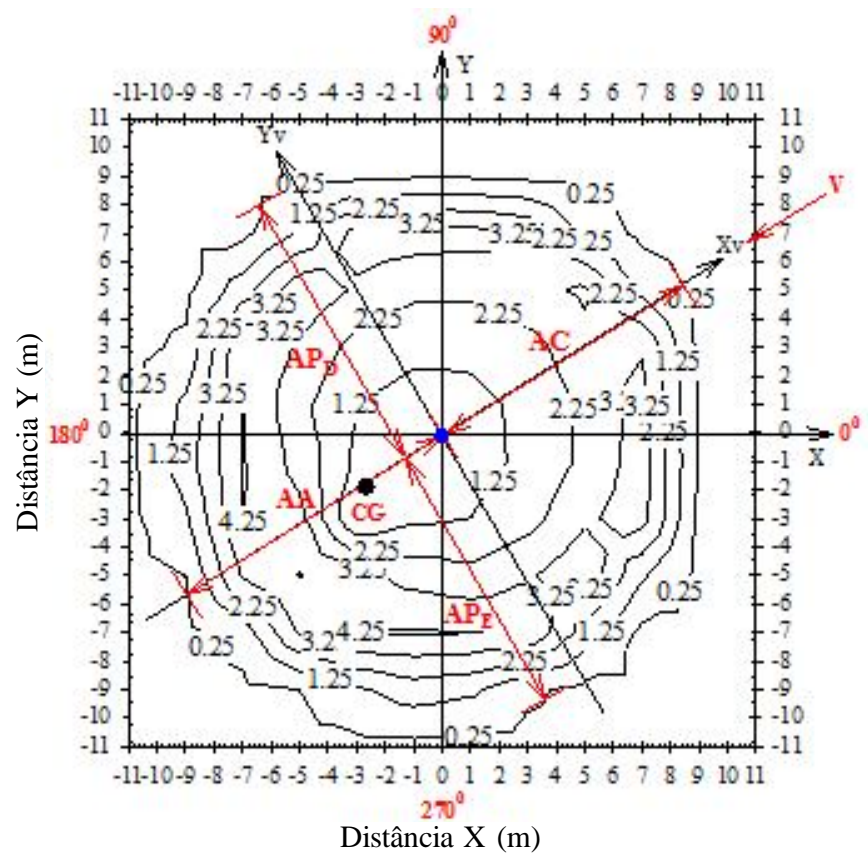

Figura 1. Inversão do eixo orientado no teste de campo $(X, Y)$ para o eixo orientado de acordo com a direção predominante do vento $(\mathrm{Xv}, \mathrm{Yv})$

Na determinação das dimensões da área molhada distorcida pelo vento foi utilizado um sistema de eixos (Xv, Yv) orientados pela direção predominante do vento, durante os ensaios. $\mathrm{O}$ sentido predominante do vento foi definido na direção da reta, que liga a posição de instalação do aspersor $(X=0, Y=0)$ ao centro de gravidade $(X=X G, Y=Y G)$ da distribuição espacial de água (Figura 1).

$\mathrm{Na}$ determinação das coordenadas $(\mathrm{XG}, \mathrm{YG})$ do centro de gravidade (CG) da distribuição espacial de água do aspersor, observada em cada ensaio, utilizaram-se as coordenadas dos 144 coletores, juntamente com os valores das intensidades de precipitação $\left(I_{i, j}\right)$ em cada coletor, de acordo com as equações:

$$
\begin{aligned}
& X G=\frac{\sum_{i=1}^{i=12} \sum_{j=1}^{j=12} X_{i, j} \cdot I_{i, j}}{\sum_{i=1}^{i=12} \sum_{j=1}^{j=12} I_{i, j}} \\
& Y G=\frac{\sum_{i=1}^{i=12} \sum_{j=1}^{j=12} Y_{i, j} \cdot I_{i, j}}{\sum_{i=1}^{i=12} \sum_{j=1}^{j=12} I_{i, j}}
\end{aligned}
$$

em que:

$$
\begin{aligned}
& X_{i, j} \text { - coordenada } X \text { do coletor } A(i, j) \\
& Y_{i, j} \text { - coordenada } Y \text { do coletor } A(i, j)
\end{aligned}
$$

As medidas das distâncias do aspersor até a extremidade da área molhada pelo vento, definidas pela posição da isoieta, correspondentes a uma intensidade de precipitação de 0,25 $\mathrm{mm} \mathrm{h}^{-1}$, foram determinadas em quatro direções de alcance: (i) a favor ao vento (AA); (ii) contra o vento (AC); (iii) perpendicular à direita $\left(\mathrm{AP}_{\mathrm{D}}\right) \mathrm{e}(\mathrm{iv})$ perpendicular à esquerda $\left(\mathrm{AP}_{\mathrm{E}}\right)$ em relação ao vento; posteriormente, os valores da média dos alcances perpendiculares $\left(\mathrm{AP}=\left(\mathrm{AP}_{\mathrm{D}}+\mathrm{AP}_{\mathrm{E}}\right) / 2\right)$ e os alcances contra (AC) e a favor (AA) a direção do vento, foram expressos em termos de porcentagem (100AP/R, 100AC/R, $100 \mathrm{AA} / \mathrm{R})$ do alcance sem vento $(\mathrm{R})$ do aspersor.

$\mathrm{Na}$ modelagem das dimensões da área molhada distorcida pelo vento foi utilizado o modelo semiempírico de Richards \& Weatherhead (1993), o qual utiliza o sistema de eixos $\left(\mathrm{X}_{\mathrm{v}}, \mathrm{Y}_{\mathrm{v}}\right)$ orientado em relação à direção do vento, conforme esquematizado na Figura 1, sendo a relação entre as coordenadas de um ponto de impacto da água no solo que ocorre na condição de ausência de vento $\left(\mathrm{X}_{\mathrm{v} 0}, \mathrm{Y}_{\mathrm{v} 0}\right)$ e as coordenadas de impacto deste mesmo ponto sob condições de vento $\left(\mathrm{X}_{\mathrm{v}}, \mathrm{Y}_{\mathrm{v}}\right)$ expressas, de acordo com Richards \& Weatherhead (1993), pelas seguintes equações:

$$
\begin{aligned}
& x_{v}=x_{v 0}-\left[A+B \cdot\left(\frac{r}{R}\right)+C \cdot\left(\frac{r}{R}\right)^{2}\right] \cdot V- \\
&-\left[D \cdot\left(\frac{r}{R}\right)+E \cdot\left(\frac{r}{R}\right)^{2}+F \cdot\left(\frac{r}{R}\right)^{3}\right] \cdot V \cdot S \cdot \cos \theta \\
& y_{v}=y_{v 0}-\left[D \cdot\left(\frac{r}{R}\right)+E \cdot\left(\frac{r}{R}\right)^{2}+F \cdot\left(\frac{r}{R}\right)^{3}\right] \cdot V \cdot S \cdot \operatorname{sen} \theta
\end{aligned}
$$

em que:

A, B, C, D, E e F - constantes empíricas do modelo, m por m $\mathrm{s}^{-1}$

$\mathrm{V}$ - velocidade do vento, $\mathrm{m} \mathrm{s}^{-1}$

e - ângulo de lançamento do jato de água $\left(12^{0}\right.$ para os aspersores estudados).

$$
\begin{gathered}
r=\sqrt{\left(x_{\mathrm{v} 0}\right)^{2}+\left(\mathrm{y}_{\mathrm{v} 0}\right)^{2}} \\
\cos \theta=\frac{\mathrm{x}_{\mathrm{v} 0}}{\mathrm{r}}
\end{gathered}
$$

$$
\operatorname{sen} \theta=\frac{\mathrm{y}_{\mathrm{v} 0}}{\mathrm{r}}
$$

$$
\mathrm{S}=\sqrt{\operatorname{sen}^{2} \mathrm{e} \cdot \cos ^{2} \theta+\operatorname{sen}^{2} \theta}
$$


Nas Eqs. 3 e 4 os termos $\left(\mathrm{A}+\mathrm{B}(\mathrm{r} / \mathrm{R})+\mathrm{C}(\mathrm{r} / \mathrm{R})^{2}\right)$ e $(\mathrm{D}(\mathrm{r} / \mathrm{R})+$ $\left.\mathrm{B}(\mathrm{r} / \mathrm{R})^{2}+\mathrm{C}(\mathrm{r} / \mathrm{R})^{3}\right)$ representam, respectivamente, a deriva (Dv) e a redução do alcance $(\mathrm{Ra})$ devido à interrupção do fluxo de ar junto ao jato de água. Nessas equações, a função "S" fornece o seno do ângulo entre o vetor velocidade do vento e a direção do jato, a partir do ângulo do jato no plano vertical (e em graus) e do ângulo entre o vetor vento e o jato de água no plano horizontal ( $\theta$ em graus).

A partir das Eqs. 3 e 4 e de acordo com o esquematizado na Figura 2, foi possível obter as equações que determinam as relações entre velocidade do vento e os alcances até a extremidade da área molhada pelos aspersores de tamanho médio modelos Agropolo/NY e Naan/5024, tomada nas quatro direções consideradas: (i) a favor do vento (AA); (ii) contra o vento $(\mathrm{AC})$; (iii) perpendicular à direita $\left(\mathrm{AP}_{\mathrm{D}}\right)$; e (iv) perpendicular à esquerda $\left(\mathrm{AP}_{\mathrm{E}}\right)$ do vento.

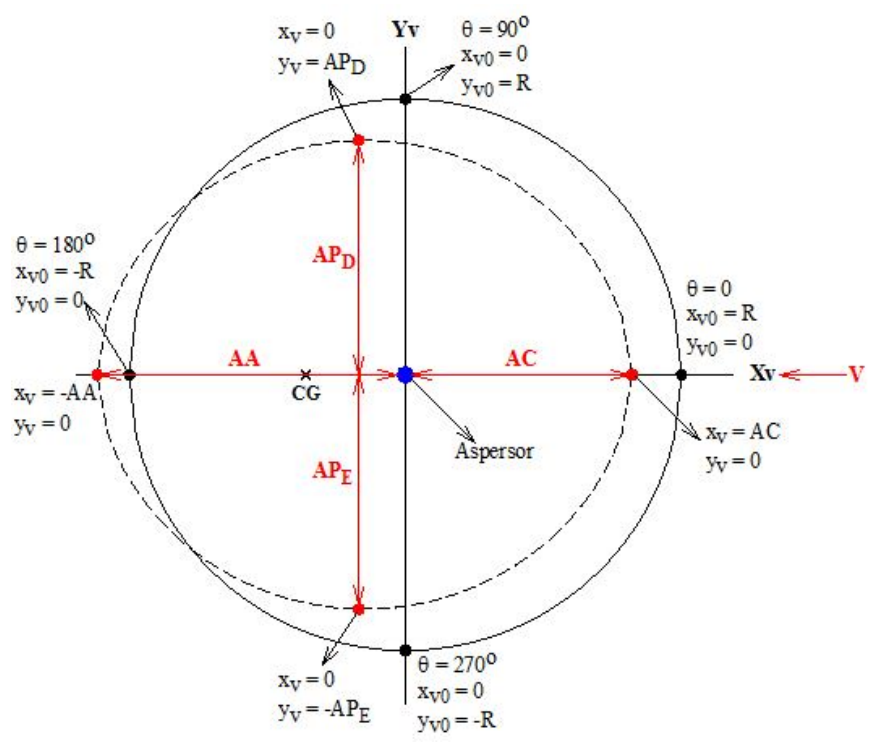

Figura 2. Distorção das dimensões do padrão de distribuição de água do aspersor devido à incidência do vento

Conforme esquematizado na Figura 2 e seguindo os mesmos critérios descritos por Oliveira et al. (2009) considerando, porém, o ângulo de inclinação de saída do jato de água igual a $12^{\circ}$, que resulta em um valor de $S=0,208$ nos alcances a favor e contra o vento e um valor unitário nas direções perpendiculares ao vento, obtidos pela Eq. 8, os alcances a favor (AA), contra (AC) e perpendiculares ao vento $(\mathrm{AP}=$ $\left.\left(\mathrm{AP}_{\mathrm{D}}+\mathrm{AP}_{\mathrm{E}}\right) / 2\right)$ podem ser expressos em termos de porcentagem do alcance sem vento do aspersor $(\mathrm{R})$ pelas equações:

$$
\begin{gathered}
100 \cdot \frac{\mathrm{AA}}{\mathrm{R}}=100+100 \cdot\left[\frac{(\mathrm{A}+\mathrm{B}+\mathrm{C})}{\mathrm{R}}-0,208 \cdot \frac{(\mathrm{D}+\mathrm{E}+\mathrm{F})}{\mathrm{R}}\right] \cdot \mathrm{V} \\
100 \frac{\mathrm{AC}}{\mathrm{R}}=100-100 \cdot\left[\frac{(\mathrm{A}+\mathrm{B}+\mathrm{C})}{\mathrm{R}}+0,208 \cdot \frac{(\mathrm{D}+\mathrm{E}+\mathrm{F})}{\mathrm{R}}\right] \cdot \mathrm{V} \\
100 \cdot \frac{\mathrm{AP}}{\mathrm{R}}=100-100 \cdot \frac{(\mathrm{D}+\mathrm{E}+\mathrm{F})}{\mathrm{R}} \cdot \mathrm{V}
\end{gathered}
$$

As Eqs. 9, 10 e 11 permitem estabelecer, por meio dos coeficientes angulares da reta ajustada aos pares de valores experimentais, as estimativas dos valores das somas dos coeficientes empíricos (100(D/R + E/R + F/R) e $100(\mathrm{~A} / \mathrm{R}+\mathrm{B} / \mathrm{R}$ $+\mathrm{C} / \mathrm{R})$ ) do modelo de Richards \& Weatherhead (1993).

\section{RESULTADOS E DISCUSSÃO}

A viabilidade da utilização no modelo semiempírico de Richards \& Weatherhead (1993), de valores normalizados dos coeficientes empíricos (A/R, B/R, C/R, D/R, E/R, F/R) é demonstrada na Figura 3 em que, pela análise da distribuição
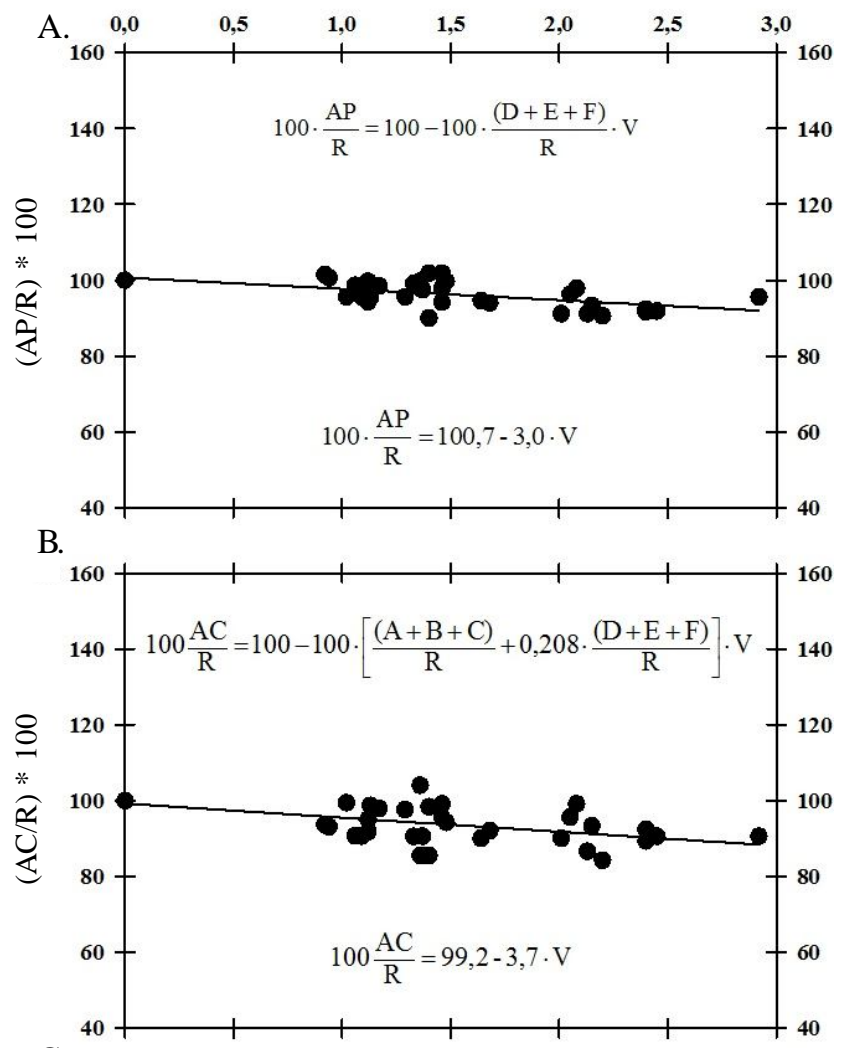

C.

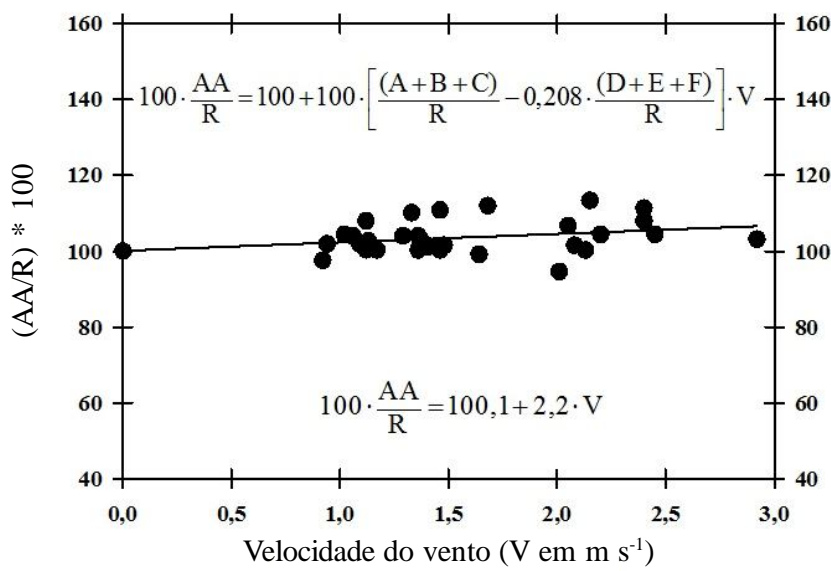

Figura 3. Alcances relativos até a extremidade da área molhada pelo aspersor, tomados nas direções: $(A)$ : perpendicular (AP), (B) contrária $(A C)$ e $(C)$ a favor (AA) ao vento, sob diferentes velocidades de vento 
dos pontos experimentais é demonstrada, para aspersores de tamanho médio, a adequação da hipótese de uma relação linear entre alcance do aspersor e velocidade do vento.

Para cada uma das três curvas (AP, AC e AA) apresentadas na Figura 3 foram considerados 32 valores de distâncias determinadas com velocidades de vento maiores que $0,9 \mathrm{~m} \mathrm{~s}^{-1}$, dos aspersores modelos Agropolo/NY e Naan/5024 e 6 valores de distâncias determinadas na ausência de vento. Esses conjuntos de dados cobrem uma variação no valor do alcance sem vento do aspersor Agropolo/NY que abrange desde $10,60 \mathrm{~m}$ (pressão de $194 \mathrm{kPa}$ ) até $11,70 \mathrm{~m}$ (pressão de $294 \mathrm{kPa}$ ) e de 9,63 m (pressão de $245 \mathrm{kPa}$ ) até 10,25 m (pressão de $343 \mathrm{kPa}$ ) para o aspersor modelo Naan/5024.

Conforme a Figura 3A, na direção dos alcances perpendiculares (AP) ao vento não ocorrem perdas por deriva $\left(\mathrm{Dv}=\mathrm{A}+\mathrm{B}(\mathrm{r} / \mathrm{R})+\mathrm{C}(\mathrm{r} / \mathrm{R})^{2}\right)$ mas apenas redução no alcance em virtude da interrupção do fluxo de ar junto ao jato de água $\left(\mathrm{Ra}=\mathrm{D}(\mathrm{r} / \mathrm{R})+\mathrm{B}(\mathrm{r} / \mathrm{R})^{2}+\mathrm{C}(\mathrm{r} / \mathrm{R})^{3}\right)$, comprovada pela Eq. 11. Todavia, nos alcances contrário $(\mathrm{AC})$ e a favor (AA) à direção do vento (Figuras 3B e 3C, respectivamente) ambos os fenômenos, Dv e Ra, ocorrem simultaneamente, como descrito pelas Eqs. 9 e 10 e observado por Richards \& Weatherhead (1993).

A Figura 3A mostra a variação do valor médio $\left(\mathrm{AP}=\left(\mathrm{RP}_{\mathrm{D}}+\mathrm{RP}_{\mathrm{E}}\right) / 2\right)$ dos alcances entre o aspersor e a extremidade da área molhada, em função da velocidade do vento, tomada perpendicularmente à direção do vento. Apesar do baixo valor obtido na regressão do modelo linear $\left(\mathrm{R}^{2}=0,42\right)$, há uma proximidade entre o valor assumido pelo intercepto da reta ajustado ao valor teórico esperado (100,7 e 100), sendo este um indicador da adequação do uso de parâmetros empíricos normalizados. $\mathrm{O}$ confronto entre os termos das duas equações apresentadas na Figura 3A, fornece uma primeira estimativa do valor da soma dos coeficientes empíricos normalizados utilizados no cálculo da redução do alcance devido à interrupção do fluxo de ar induzido (D/R + E/R + F/R =0,030 $\left.\mathrm{s} \mathrm{m}^{-1}\right)$.

$A$ variação em função do valor da velocidade do vento, do valor do alcance relativo entre o aspersor e a extremidade da área molhada tomada na direção contrária ao vento (AC/R), é mostrada na Figura 3B. A proximidade entre o valor assumido pelo intercepto da reta ajustado e o valor teórico esperado (99,2 e 100) é um indicador da adequação do uso de parâmetros empíricos normalizados, porém não se obteve um bom ajuste dos dados observados no modelo linear $\left(R^{2}=0,32\right)$. Oliveira et al. (2009) também obtiveram, em estudos com o canhão hidráulico Plona-RL250, boa proximidade entre o valor assumido pelo intercepto da reta e o valor teórico ajustado, mas com um ajuste melhor dos dados observados no modelo linear, $\mathrm{R}^{2}=$ 0,85 e $\mathrm{R}^{2}=0,74$ para os alcances perpendicular e contra o vento, respectivamente.

Com base no confronto entre as duas equações mostradas na Figura $3 \mathrm{~B}$ e o valor previamente estimado de $\mathrm{D} / \mathrm{R}+\mathrm{E} / \mathrm{R}+\mathrm{F} /$ $\mathrm{R}\left(0,030 \mathrm{~s} \mathrm{~m}^{-1}\right)$, pode-se estabelecer uma primeira estimativa do valor da soma dos coeficientes empíricos normalizados, utilizados no cálculo da deriva pelo vento $(\mathrm{A} / \mathrm{R}+\mathrm{B} / \mathrm{R}+\mathrm{C} / \mathrm{R}=$ $\left.0,031 \mathrm{~s} \mathrm{~m}^{-1}\right)$.

Em função do valor da velocidade do vento, do valor da distância relativa entre o aspersor e a extremidade da área molhada, a variação tomada na mesma direção do vento (AA/ R) é mostrada na Figura 3C, caso em que, tal como obtido por Oliveira et al. (2009), o coeficiente de determinação da reta de ajuste foi baixo $\left(\mathrm{R}^{2}=0,16\right)$, porém a proximidade entre o valor assumido pelo intercepto da reta ajustado e o valor teórico esperado (100,1 e 100) foi mantida.

A forma ascendente da reta de ajuste (Figura 3C) indica que, para o alcance medido na mesma direção do vento, o efeito de redução do alcance (Ra), provocado pela interrupção do fluxo de ar, é pouco menor que o efeito da deriva (Dv). Com base no confronto entre as duas equações mostradas na Figura $3 \mathrm{C}$ e no valor previamente estimado de $\mathrm{D} / \mathrm{R}+\mathrm{E} / \mathrm{R}+\mathrm{F} / \mathrm{R}(0,030$ $\mathrm{s} \mathrm{m}^{-1}$ ), é possível estabelecer uma segunda estimativa para o valor da soma dos fatores empíricos utilizados no cálculo da deriva pelo vento: $\left(\mathrm{A} / \mathrm{R}+\mathrm{B} / \mathrm{R}+\mathrm{C} / \mathrm{R}=0,028 \mathrm{~s} \mathrm{~m}^{-1}\right)$. Desta forma, assume-se, pela proximidade dos valores obtidos, o valor médio entre a primeira e a segunda estimativa para o cálculo da deriva pelo vento $\left(\mathrm{A} / \mathrm{R}+\mathrm{B} / \mathrm{R}+\mathrm{C} / \mathrm{R}=0,0295 \mathrm{~s} \mathrm{~m}^{-1}\right)$.

Com base nos resultados apresentados na Figura 3, podese afirmar que: (i) na direção perpendicular ao vento (AP) o alcance do aspersor sofre redução de 3,0\% em referência ao alcance sem vento $(\mathrm{R})$, para cada incremento de $1,0 \mathrm{~m} \mathrm{~s}^{-1}$ na velocidade do vento; (ii) na direção contrária ao vento $(\mathrm{AC}) \mathrm{o}$ alcance é reduzido em $3,7 \%$ para cada $1,0 \mathrm{~m} \mathrm{~s}^{-1}$ de incremento na velocidade do vento e (iii) na direção a favor ao vento (AA) o alcance do aspersor aumenta $2,2 \%$ em relação ao alcance sem vento $(\mathrm{R})$ para cada $1,0 \mathrm{~m} \mathrm{~s}^{-1}$ de incremento na velocidade do vento.

Os valores de distorção do padrão espacial de distribuição de água obtidos neste estudo são inferiores aos obtidos por Tarjuelo et al. (1999) na avaliação dos aspersores de tamanho médio Agros35® e Rain Bird 46H; este fato pode ser atribuído aos menores valores de velocidade de vento que compõem o intervalo considerado no estudo ( 0 a $\left.3 \mathrm{~m} \mathrm{~s}^{-1}\right)$, quando comparado com o desses autores $\left(0\right.$ a $\left.8 \mathrm{~m} \mathrm{~s}^{-1}\right)$.

Devido à velocidade e à direção do vento, a porcentagem da variação no alcance, para os aspersores de tamanho médio modelos Agropolo/NY e Naan/5024 se diferenciou dos valores obtidos para canhões hidráulicos, por Richards \& Weatherhead (1993) e Oliveira et al. (2009), nas direções de alcance a favor (AA), contra (AC) e perpendicular (AP) ao vento, conforme mostrado na Tabela 2. Ainda assim, o alcance medido na mesma direção do vento (AA) aumenta em menores proporções, quando comparado com o das reduções ocorridas nas direções contra (AC) e perpendicular (AP) ao vento, estando este comportamento de acordo com o previsto por Richards \& Weatherhead (1993) e comprovado por Oliveira et al. (2009), para canhões hidráulicos, e por Tarjuelo et al. (1999), para aspersores de tamanho médio.

A maior distorção pela ação do vento da área molhada por canhões hidráulicos em comparação à área molhada por aspersores de tamanho médio, é contraditória com a literatura. De acordo com Playán et al. (2005), gotas maiores são mais resistentes à deriva pelo vento, visto que gotas maiores apresentam menor área por unidade de massa e, consequentemente, são menos afetadas pelo vento; apesar disto, devese considerar que o canhão Plona-RL250 operou com maiores velocidades de vento (valores próximos de $5,0 \mathrm{~m} \mathrm{~s}^{-1}$ ) que os 
Tabela 2. Variação do alcance devido à velocidade do vento, nas direções, a favor (AA), contra (AC) e perpendicular (AP), para aspersores de tamanho médio (Agropolo/NY e Naan/5024) e para canhões hidráulicos

\begin{tabular}{lccc}
\hline \multicolumn{1}{c}{ Autor/Aspersor } & AA & AC & AP \\
\cline { 2 - 4 } & & $\left(\%\right.$ por $\left.\mathbf{~ m ~ s}^{-1}\right)$ & \\
Agropolo/NY e Naan/5024 & 2,2 & $-3,7$ & $-3,0$ \\
$\begin{array}{l}\text { Oliveira et al. (2009) / } \\
\begin{array}{l}\text { Plona - RL250 } \\
\text { Richards \& Weatherhead } \\
\text { (1993) / Nelson P150 }\end{array}\end{array}$ & 1,3 & $-6,3$ & $-7,3$ \\
\hline
\end{tabular}
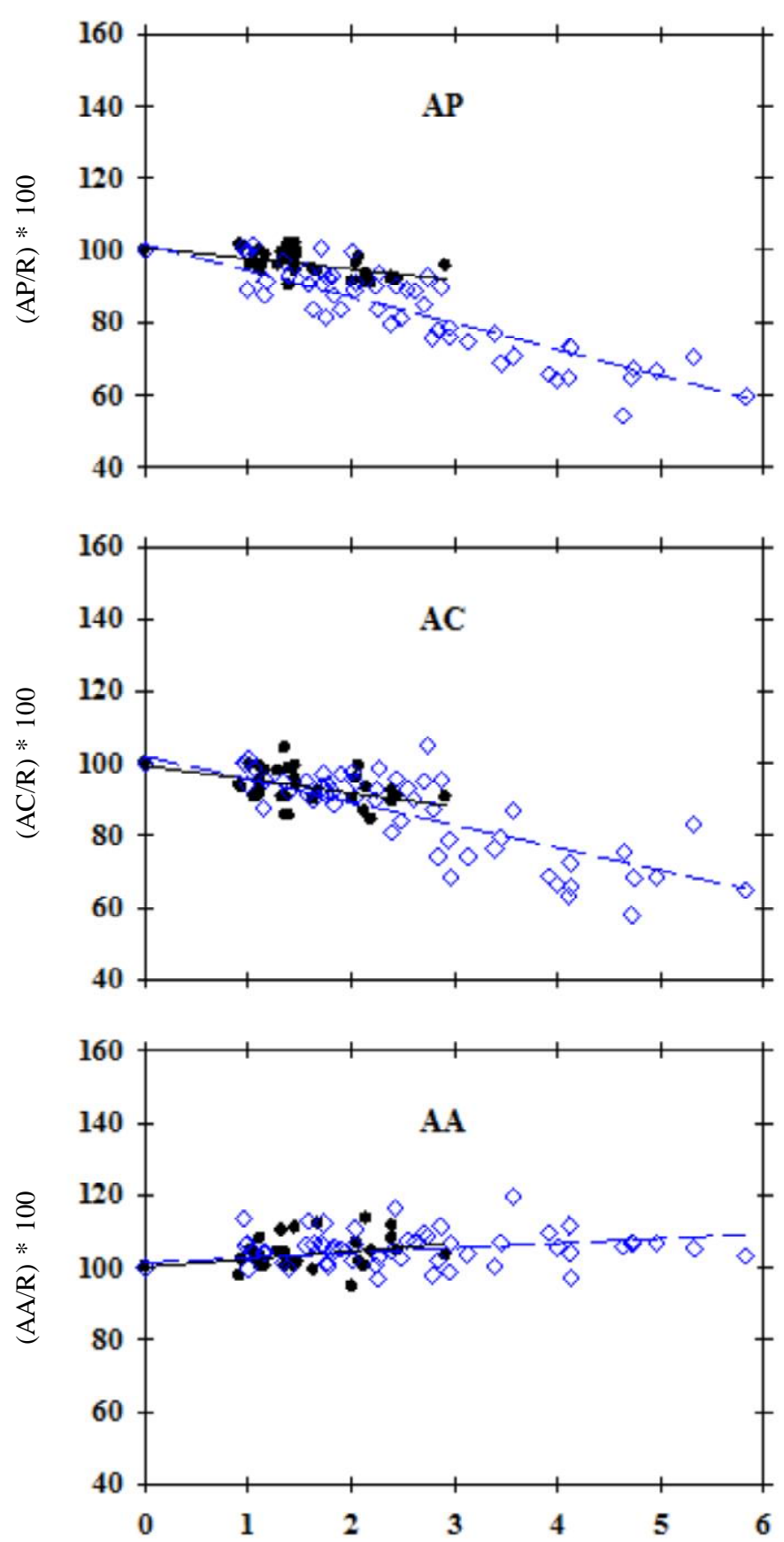

aspersores de tamanho médio (cujas velocidades de vento não excederam 3,0 $\mathrm{m} \mathrm{s}^{-1}$ ) conforme observado na coluna da esquerda Figura 4, que compara os 38 valores de alcance do jato dos aspersores de tamanho médio modelos Agropolo/NY e Naan/ 5024 (ângulo de saída do jato de $12^{\circ}$ ) com os 79 valores de alcance do jato do aspersor Plona-RL250 (ângulo de saída do jato de $24^{\circ}$ ) analisados por Oliveira et al. (2009). Comparando apenas os valores dos ensaios do canhão Plona-RL250 em condições de vento de até $3,0 \mathrm{~m} \mathrm{~s}^{-1}$ (coluna direita da Figura 4) os valores de variação do alcance analisados (AA, AC e AP) foram próximos aos obtidos com os aspersores de tamanho médio avaliados neste estudo.
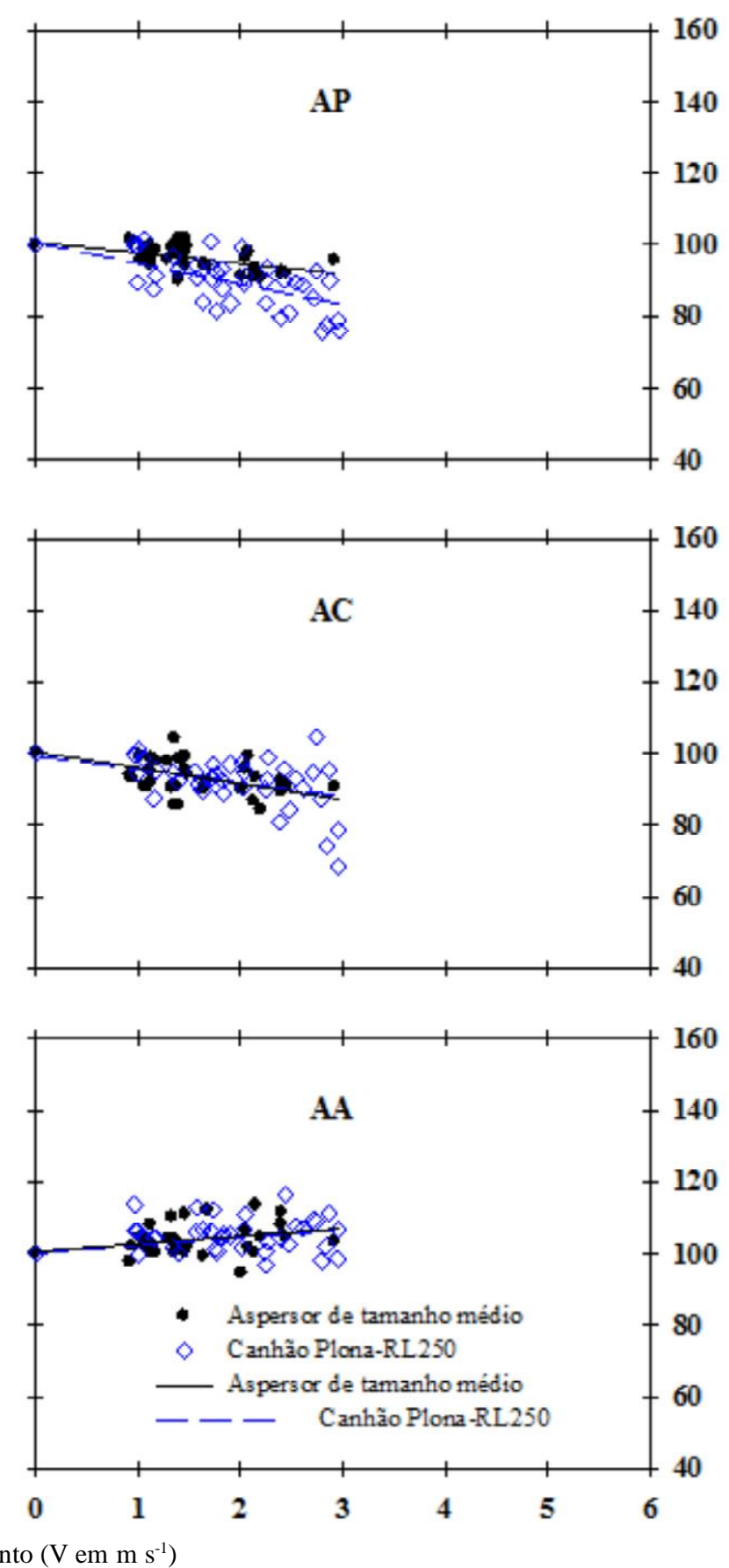

Figura 4. Comparação das variações do alcance a favor (AA), contra (AC) e perpendicular (AP) à direção do vento, entre o canhão hidráulico Plona-RL250 e aspersores de tamanho médio modelos Agropolo/NY e Naan/5024 
Além disso, é oportuno considerar que em canhões hidráulicos existe uma interação significativa entre as gotas e o ar circunvizinho. De acordo com Richards \& Weatherhead (1993), na simples teoria de trajetórias de gotas considera-se que o movimento de ar não é afetado pela transferência de momento da água para o ar; entretanto, para canhões hidráulicos essa trajetória é significativa e o jato de água induzirá o ar circunvizinho a se mover com ele. Tal movimento de ar retarda o jato e reduz o arraste de gotas individuais, obtendo-se maiores reduções no alcance.

Observa-se também (Figura 4) que na direção perpendicular ao vento (AP) ocorreu maior variação no alcance entre aspersores de tamanho médio avaliados e o canhão PlonaRL250 do que nas direções contra (AC) e a favor (AA) ao vento. De acordo com Richards \& Weatherhead (1993), isto ocorre porque a redução na direção perpendicular ao vento (AP) é consequência apenas do rompimento do fluxo de ar induzido $\left(\mathrm{Ra}=\mathrm{D}(\mathrm{r} / \mathrm{R})+\mathrm{B}(\mathrm{r} / \mathrm{R})^{2}+\mathrm{C}(\mathrm{r} / \mathrm{R})^{3}\right)$, que é mais relevante em canhões hidráulicos do que em aspersores de tamanho médio.

Deve-se levar em consideração, ainda, que a velocidade do vento nos ensaios com o canhão Plona-RL250 foi mensurada a uma altura de $2 \mathrm{~m}$ da superfície do solo porém a altura do jato de água em canhões hidráulicos geralmente atinge valores superiores a $2 \mathrm{~m}$. A velocidade do vento aumenta em escala logarítmica à medida em que a altura também aumenta sendo necessário, então, fazer uma correção nos valores mensurados de velocidade de vento quando medidos a $2 \mathrm{~m}$ da superfície do solo para se obter a velocidade real de vento a uma altura superior a 2 m, como sugerido por Allen et al. (1998), o que levaria, provavelmente, a valores ainda mais próximos das variações de alcance a favor (AA), contra (AC) e perpendiculares (AP) à direção do vento, entre os aspersores de tamanho médio Agropolo/NY e Naan/5024 e o canhão PlonaRL250.

\section{Conclusão}

A hipótese da existência de uma relação linear entre o alcance do jato de aspersores e a velocidade do vento, já comprovada para aspersores de alta pressão (canhões hidráulicos), foi comprovada para aspersores de tamanho médio.

\section{LITERATURA CITADA}

Allen, R. G.; Pereira, L. S.; Raes, D.; Smith, M. Guidelines for computing crop water requeriments. Rome: FAO, 1998. 308p. FAO Irrigation and Drainage, 56

Bernuth, R. D. von; Seginer, I. Wind considerations in sprinkler system design. Visions of the future. In: National Irrigation Symposium, 3, 1990, Phoenix. Proceedings... Phoenix: ASAE, 1990. CD Rom
Beskow, S.; Faria, L. C.; Colombo, A.; Moura, D. C. Modelagem das perdas de água por evaporação e arraste em aspersores de média pressão. Revista Brasileira de Engenharia Agrícola e Ambiental, v.15, p. 221-228, 2011.

Carrión, P.; Tarjuelo, J. M; Montero, J. SIRIAS: A simulation model for sprinkler irrigation. Part I: Description of model. Irrigation Science, v.20, p.73-84, 2001.

Faria, L. C.; Colombo, A.; Oliveira, H. F. E. de; Prado, G. Simulação da uniformidade da irrigação de sistemas convencionais de aspersão operando sob diferentes condições de vento. Engenharia Agrícola, v.29, p.19-27, 2009.

Granier, J.; Molle, B.; Deumier, J. M. IRRIPARC-Part 1: Modeling spatial water distribution under a sprinkler in windy conditions. ICID International workshop, Montpellier, 2003. Proceedings... Aix en Provence: Cemagref, 2003. p.14-19.

ISO - International Organization for Standardization. ISO 77492. Irrigation equipment - Rotating sprinklers - Part 2: Uniformity of distribution and test methods, Switzerland: ISO, 1990.6p.

Keller, J.; Bliesner, R. D. Sprinkle and trickle irrigation. New York: Anavi Book/Van Nostrand Reinhold, 1990. 652p.

Montero, J.; Tarjuelo, J. M.; Carrión, P. SIRIAS: a simulation model for sprinkler irrigation. Part II: Calibration and validation of the model. Irrigation Science, v.20, p.85-98, 2001.

Newell, G. Traveling gun simulation model TRAVGUN: User's manual and technical documentation. Toowoomba: National Centre for Engineering in Agriculture-NCEA. University of Southern Queensland, 2003. 89p.

Oliveira, H. F. E.; Colombo, A.; Faria, L. C. Modelagem dos efeitos do vento sobre as dimensões do alcance do jato de um canhão hidráulico. Revista Brasileira de Engenharia Agrícola eAmbiental, v.13, p.818-824, 2009.

Playán, E.; Salvador, R.; Faci, J.M.; Zapata, N.; Martinez-Cob, A.; Sanches, I. Day and night wind drift and evaporation losses in sprinkler solid-sets and moving laterals. Agricultural Water Management, v.76, p.139-159, 2005.

Playán, E.; Zapata, N.; Faci, J. M.; Tolosa, D.; Lacuerva, J. L.; Pelegri, J. Salvador, R.; Sanches, I.; Lafita, A. Assessing sprinkler irrigation uniformity using a ballistic simulation model. Agricultural Water Management, v.84, p.89-100. 2006.

Prado, G.; Colombo, A. Distribuição espacial de água aplicada por equipamentos autopropelidos de irrigação - Parte I: Modelagem com o Simulasoft. Irriga, v. 15, p.51-62, 2010a.

Prado, G.; Colombo A. Distribuição espacial de água aplicada por equipamentos autopropelidos de irrigação - Parte II: Validação do Simulasoft. Irriga, v.15, p.63-74, 2010 b.

Richards, P. J.; Weatherhead, E. K. Prediction of Raingun Application Patterns in Windy Conditions. Journal of Agricultural Engineering Research, v.54, p.281-291. 1993.

Smith, R. J.; Gillies, M. H.; Newell, G.; Foley, J. P. A decision support model for travelling gun irrigation machines, Biosystems Engineering, v.100, p.126-136, 2008.

Tarjuelo, J. M.; Montero, J.; Carrión, P. A.; Honrubia, F. T.; Calvo, M. A. Irrigation uniformity with medium size sprinklers. Part II: Influence of wind and other factors on water distribution. Transactions of the American Society of Agricultural Engineers, v.42, p.677-689, 1999. 